\title{
Article \\ Correlations between Anxiety and Depression, and Mental Elasticity in Malignant Hematopathy Patients
}

\author{
Ning Cao ${ }^{1}$, Susu Yan ${ }^{1}$, Jinli Zhu ${ }^{1}$, Yan Liu ${ }^{1}$, Chuanxin Liu ${ }^{1 *}$, Ling Liu ${ }^{2}$ \\ ${ }^{1}$ Jining Medical University, Jining 272067, China; \\ ${ }^{2}$ Affiliated Hospital of Jining Medical University, Jining 272067, China.
}

${ }^{*}$ Corresponding Author: Chuanxin Liu. Email: liuchuanxin2003b @163.com.

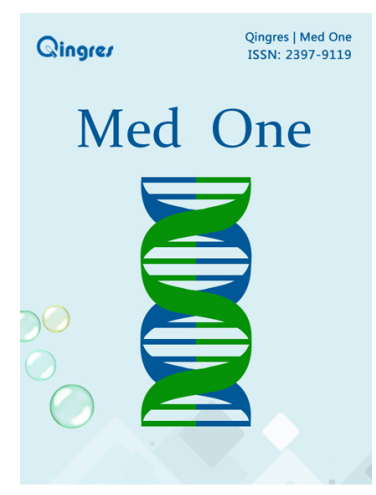

https://mo.qingres.com

\section{GOPEN ACCESS}

DOI: $10.20900 / \mathrm{mo} .20180006$

Received: May 28, 2018

Accepted: August 24, 2018

Published: August 30, 2018

Copyright: $\odot 2018$ by the authors, Licensee Qingres, London, United Kingdom. This is an open access article distributed under the terms and conditions of the Creative Commons Attribution License (CC BY 4.0, https://creativecommons.org/licenses/by/4.0/).

\begin{abstract}
Objective: To explore the relationship between: anxiety and depression, and mental elasticity in malignant hematopathy patients.

Methods: Two hundred and eighty-four malignant hematopathy patients were studied using: a demographic questionnaire; resilience scale scores; self-rating anxiety scale scores; and, self-rating depression scale scores. The statistical analysis was performed using SPSS17.0.

Results: Patients with malignant hematopathy had anxiety and depression scores significantly higher than the national norm and were statistically significant. Resilience scores, and their dimensions, of a group of patients positive for anxiety and depression were significantly lower than those of a group of patients negative for anxiety and depression, which was statistically significant $(p<0.01)$. Mental elasticity scores, and their dimensions, negatively correlated with anxiety and depression levels. A regression analysis of optimism and strength, negatively predicted depression, while optimism negatively predicted anxiety.
\end{abstract}

Conclusion: Patients with malignant hematopathy have low mental elasticity which closely relates to anxiety and depression. Mental elasticity is a predictor of both anxiety and depression.

Keywords: malignant hematopathy; resilience scale; anxiety; depression 


\section{INTRODUCTION}

Similar to the most solid tumors, malignant hematopathy $(\mathrm{MH})$ incidence rates are rising ${ }^{[1]}$. As medical research matures, $\mathrm{MH}$ cure rates have improved. $\mathrm{MH}$ appears insidiously as do most refractory diseases, which greatly impacts patient physical and mental health. Studies have shown that $\mathrm{MH}$ patient anxiety and depression incidence rates, influenced by fear of disease and prognosis, are higher than that for non-MH sufferers ${ }^{[2,3]}$. Recently, mental elasticity (ME) or resilience, has become a research subject undergoing intense study in the field of psychology. Some research results show that better ME can significantly improve a patients' ability to resist stress and impede or prevent negative mental health from developing ${ }^{[4]}$. This study explores the relationships between $\mathrm{MH}$ patient ME, and anxiety and depression in order to improve patient ME so as assist them in overcoming psychological damage.

\section{SUBJECTS AND METHODS}

\subsection{Subjects}

Between January and June of 2017, patients with schizophrenia who had been hospitalized and treated for 12 months in the Affiliated Hospital of Jining Medical University Hematopathy Department, were enrolled.

Inclusion Criteria: a. patients in compliance with the diagnostic criteria for $\mathrm{MH}$, including, Iymphoma, multiple myeloma, acute myeloid leukemia, acute and chronic leukemia, acute promyelocytic leukemia, non-Hodgkin's lymphoma, follicular lymphoma, chronic myeloid leukemia, and other related diseases ${ }^{[5]}$; b. who were able to communicate effectively; c. with a certain ability to understand and read; and, d. chose to voluntary participate in this study. Exclusion criteria: a. patients with an unstable, or rapidly deteriorating, condition; $b$. patients unwilling to participate in this study. This study obtained patient and their legal guardian's consents and approval of the Affiliated Hospital of Jining Medical University Ethics Committee.

\subsection{Research methods}

Questionnaire survey was adopted in this study. Three hundred questionnaires were distributed. Two hundred and eighty-four valid questionnaires were returned. The effective rate was $94.67 \%$.

\subsubsection{Questionnaires for general information}

It included, among other things, age, gender, marriage status, education level, personal income, household registration location, working status, children situation, time of illness.

\subsubsection{Connor-Davidson resilience scale (CD-RISC)}

The original scale consisted of 25 items which were rated on Richter's 5-point scale, with 0-4 indicating: Not All True; Rarely True; Sometimes True; Often True; and, True Nearly All the Time, respectively. This scale was co-developed by Connor and Davidson, psychologists in the United States. It is now widely used ${ }^{[6]}$. The highest score of the scale is 100 . The higher the score, the greater patient mental resilience is. This scale was also translated into Chinese. The CD-RISC mental resilience scale in its Chinese version has three factors: resilience; strength; and optimism. A three-dimensional method was used: the resilience measure included items 11 , $12,13,14,15,16,17,18,19,20,21,22$, and 23; strength included items $1,5,7,9,10,24$, and 25; and, optimism included items 2, 3, 4, and 6 .

\subsubsection{Self-rating depression scale (SDS)}

The SDS scale has 20 items. The score is an integer obtained according to symptom occurrence frequency. In China, those with an SDS standard score equal to, or greater than 50 , are deemed to suffer from a depressive symptom. According to Chinese norm results, the SDS scale had four ranks: a little of the time; some of the time; a good part of the time; and, most of the time. Positive scores were rated as raw scores of $1,2,3$, and 4 , respectively. After assessment ended, raw scores for the 20 items were totaled to obtain a total raw score. A standard score was obtained by rounding the product of the total raw score and multiplying by 1.25 . The SDS total raw score normal upper limit was 41 points. A lower value represents a less depressive patient. Depression was diagnosed when the score was equal to, or greater than 50 .

\subsubsection{Self-rating anxiety scale (SAS)}

The SAS scale had 20 items. There were four ranks: a little of the time; some of the time; a good part of the time; and, most of the time according to the frequency of symptoms defined by the items. Positive scores were rated as raw scores of 1, 2, 3, and 4 points, respectively. According to the Chinese norm results, the cutoff value for an SAS standard score is 50 . Those whose SAS standard score was equal to, and greater than 50 were diagnosed as having a depressive disorder. 


\subsection{Statistical analysis}

A statistical analysis was performed using SPSS17.0. Descriptive analysis was available for general information or date; measurement data groups were compared using a $t$ test. Correlation analysis and regression analysis were used among variables. A score of $p<0.05$ or 0.01 indicates statistical significance.

\section{RESULTS}

\subsection{Anxiety and depression in $\mathrm{MH}$ patients}

Table 1 shows that, compared to the national norm, anxiety scores $(t=20.93, p=0.000)$ and depression scores $(t=20.17, p=0.000)$ in $\mathrm{MH}$ patients were significantly higher than the national norm ${ }^{[7,8]}$ and were statistically significant.

\subsection{Statistical analysis of mental resilience for $284 \mathrm{MH}$ patients}

The ME score of all MH patients was $69.96 \pm$ 15.32 , including a resilience score of $25.33 \pm 8.71$, a strength score of $14.31 \pm 5.03$, and an optimism score of $9.35 \pm 2.89$.

\subsection{Comparison of mental resilience of MH patients}

Those whose anxiety and depression scores of 50 were divided into two groups: (1) a positive group; (2) a negative group. The positive group is patients with either a positive SAS or a positive SDS score. The remaining patients were included in the negative group.

Table 2 shows that the various dimension scores for the two positive groups were each lower than for the negative group $(t=-4.16, p=0.00 ; t=-4.21$, $p=0.000 ; t=-4.45, p=0.000 ; t=-4.10, p=0.00$ ) and were statistically significant.

\subsection{Correlation analysis of ME and anxiety and depression of MH patients (Pearson correlation)}

Table 3 shows that the correlation analysis of ME and anxiety and depression of $\mathrm{MH}$ patients were significantly negatively correlated on ME and various dimension scores for anxiety and depression $(p<0.01)$.

\subsection{Regression analysis of anxiety, depression and ME and various dimensions for 284 MH patients}

In the regression model of depression, strength and optimism could negatively predict depression, while optimism negatively predicted anxiety in the regression model of anxiety. See Table 4 for details.

Table 1. Anxiety and Depression Scores in MH Patients $(\overline{\mathbf{x}}+\mathbf{s})$

\begin{tabular}{lcccc}
\hline Classification & Total & National Norm & $\boldsymbol{t}$ & $\boldsymbol{p}$ \\
\hline Anxiety & $43.71 \pm 11.22$ & $29.78 \pm 10.07$ & 20.93 & 0.00 \\
Depression & $42.26 \pm 111.53$ & $33.46 \pm 8.55$ & 20.17 & 0.00 \\
\hline
\end{tabular}

Table 2. Comparison of ME scores for the two Positive groups $(\overline{\mathbf{x}}+\mathbf{s})$

\begin{tabular}{llllll}
\hline Group & Case & Total score & Resilience & Strength & Optimism \\
\hline Positive group & 55 & $63.31 \pm 12.73$ & $21.33 \pm 7.72$ & $14.36 \pm 4.99$ & $8.11 \pm 2.64$ \\
Negative group & 228 & $71.63 \pm 15.48$ & $26.33 \pm 8.67$ & $17.88 \pm 5.95$ & $9.79 \pm 2.81$ \\
$t$ & & -4.16 & -4.21 & -4.45 & -4.10 \\
$p$ & 0.00 & 0.00 & 0.00 & 0.00 \\
\hline
\end{tabular}


Table 3. Correlation of ME and various dimension scores with anxiety and depression

\begin{tabular}{|c|c|c|c|c|c|c|}
\hline Factor & Mental elasticity & Resilience & Optimism & Strength & Anxiety & Depression \\
\hline Mental elasticity & - & $0.86^{* *}$ & $0.84^{* *}$ & $0.86^{* *}$ & $-0.26^{\star *}$ & $-0.47^{* *}$ \\
\hline Resilience & $0.86^{* *}$ & - & $0.74^{* *}$ & $0.81^{* *}$ & $-0.26^{\star *}$ & $-0.48^{* *}$ \\
\hline Optimism & $0.84^{* *}$ & $0.74^{* *}$ & - & $0.72^{* *}$ & $-0.30^{\star *}$ & $-0.48^{* *}$ \\
\hline Strength & $0.86^{\star *}$ & $0.81^{* *}$ & $0.72^{* *}$ & - & $-0.29^{* *}$ & $-0.49^{* *}$ \\
\hline Anxiety & $-0.26^{\star *}$ & $-0.26^{* *}$ & $-0.30^{* *}$ & $-0.29^{\star *}$ & - & $0.77^{* *}$ \\
\hline Depression & $-0.47^{* *}$ & $-0.48^{* *}$ & $-0.48^{* *}$ & $-0.49^{* *}$ & $0.77^{* *}$ & - \\
\hline
\end{tabular}

Note:** $p<0.01$ horizontally (bilaterally) is significantly correlated.

Table 4. Regression analysis of ME various dimension and anxiety and depression in MH patients

\begin{tabular}{llllll}
\hline Item & Variable & $\boldsymbol{\beta}$ & $\boldsymbol{\beta}^{\prime}$ & $\boldsymbol{t}$ & $\boldsymbol{p}$ \\
\hline Depression & Constant & 67.57 & - & 32.83 & 0.000 \\
& Optimism & -0.89 & -0.22 & -2.82 & 0.005 \\
& Strength & -0.38 & -0.28 & -2.16 & 0.03 \\
\multirow{2}{*}{ Anxiety } & Constant & 55.27 & - & 24.67 & 0.000 \\
& Optimism & -0.93 & -0.17 & -2.82 & 0.005 \\
\hline
\end{tabular}

Note: In the depression regression model, R2 $=0.53$ and corrected R2 $=-0.27$, and optimism and strength dimension negatively predicted with depression. In the anxiety regression model, $\mathrm{R} 2=0.31$ and corrected $\mathrm{R} 2=-0.09$, and the optimism dimension negatively predicted anxiety.

\section{DISCUSSION}

The scores of anxiety and depression of $284 \mathrm{MH}$ patients were significantly different from China's national norm. The statistically significance indicating that the incidence rate in $\mathrm{MH}$ patients was significantly higher than those in the non-MH population. This result resembles the Pei Wang findings ${ }^{[9]}$ for 53 $\mathrm{MH}$ patients with malignant hematopathy. The Li et al. study ${ }^{[10]}$ of 412 doctors and nurses in a general hospital emergency department pointed out that negative (inactive) life events correlate with anxiety and depression symptoms. These facts support a conclusion that $\mathrm{MH}$ is a stressor for these patients ${ }^{[11]}$. It tends to show that, in the face of stresses such as disease, the patient stimulated a non-specific stress response mechanism ${ }^{[12]}$ which aggravates the patient's psychological burden of and increases anxiety and depression.

This study obtained an ME score of $69.96 \pm$ 15.32 , which varies from the $59.73 \pm 9.38$ score obtained by Zhang et al. ${ }^{[13]}$ in their study of 185 cervical cancer patients. This may be correlated with 
the disease characteristics and other factors. The difference was statistically significant to a certain extent when comparing the ME negative group and the positive group. The dimension scores for the positive group are significantly lower than those of the negative group.

This shows that, in response to a stressor such as $\mathrm{MH}$, the mental health of patients with anxiety and depression was greatly affected, and it reduced their psychological tolerance, and also ME score. A similar finding appears in Ying et al.'s study ${ }^{[14]}$ of a population affected by the Wenchuan Earthquake. Higher ME levels beneficially effect PTSD and depression symptoms and buffer the impact of subjective experiences, such as worry for others, on PTSD and depression. This suggests that a high ME level reduces anxiety and depression incidence in $\mathrm{MH}$ patients.

The correlation analysis of $\mathrm{MH}$ patients included in this study shows that the ME score and various dimension scores were significantly negatively correlated with anxiety and depression $(p<0.01)$. $A$ regression analysis of $M E$ dimensions and anxiety and depression, among the predictors of depression, shows that the optimism dimension and the strength dimension negatively predicted depression. This is consistent with results obtained by Wang et al. ${ }^{[15]}$, showing negative correlation between depression degree and optimism dimension in $\mathrm{ME}$ in depression patients.

The optimism dimension, an anxiety-predictor, negatively predicts anxiety, this differs from the Huang et al. study ${ }^{[16]}$ which found that resilience and self-improvement of patients with breast cancer after chemotherapy negatively predicted anxiety.

In clinical practice, more attention could be paid to the psychological condition of patients in order to achieve physical and mental unity of treatment. This can be learned from the Fu study ${ }^{[17]}$ which found that ME training can significantly improve negative psychology states caused by surgical trauma and

\section{REFERENCES}

1. Köhler N, Mehnert A, Götze H. Psychological distress, chronic conditions and quality of life in elderly hematologic cancer patients: study protocol of a prospective study. BMC Cancer. 2017; 17: 700 . the side effects of radiotherapy and chemotherapy in breast cancer patients. More attention could be paid to the individual ME of patients to build confidence and overcome pain or disease.

This study has certain limitations related samples and region. The research sample size and region can be expanded to carry out more comprehensive research so as to achieve a greater breakthrough research progress in the future.

Research directions for the future: The samples were selected from a single general hospital-the Affiliated Hospital of Jining Medical University, and there may be a selection bias. No correlation was found between optimism and depression in studying the relationship between ME total scores and classification and anxiety and depression, which associate with the sample limitations. The investigations were aimed at all $\mathrm{MH}$ diseases and they were not specifically classified, so there might be interference. We hope that a larger sample size survey can be carried out in the future and specific types of diseases can be researched, and more accurate survey method to obtain research data can be explored, so as to further explore the relationship between anxiety and ME and its classifications.

\section{FUNDINGS}

1. National Natural Science Foundation of Shandong Province (program No.: ZR2016HM55);

2. Support fund from Jining Medical University (program No.: JY2017JS008).

\section{CONFLICTS OF INTEREST}

The authors declare that there is no conflict of interest regarding the publication of this paper.

2. Cao S, Wang Y, Zhu Y, Chang M, Yuan W, Li B, Zhou C, Hu Y. Investigation of Psychological Health Condition in Patients with Malignant Hematopathy. J Clin Med Pract. 2014; 18(12): 95-97. 
3. Tian J, Yang R, Zhang J, Zhang B. Psychological changes and countermeasures of patients with hematopathy in different periods of hospitalization. Heibei Med J. 2010; 32(4): 500.

4. Zhou X, Xue B, Wang Y, Ma X, Shi H, Wu C. Relationship between Self-efficiency, Social Support, Coping Style and Mental Health of Escort Ships Officers and Soldiers. Chin J Health Psychol. 2016; 24(6): 836-840.

5. Yao E, Lin F, Guo X. Diagnosis and Treatment of Malignant Hematopathy. Beijing: People's Military Medical Press. 2004: 1.

6. Connor KM, Davidson JR. Development of a new resilience scale: the Connor-Davidion Resilience scale (CD-RISC). Depressive Anxiety. 2003; 18(2): 76-82.

7. Zhang Y, Wang X, Liu H, Liu J, Zhang B. Adverse psychological reactions and countermeasures of armed police officers and soldiers during the prevention and treatment of atypical pneumonia. Med J Chin Peop Armed Poli Forc. 2003; 14(7): 432.

8. Wang C, Cai Z, Xu Q. Assessment analysis of 1340 normal people using Self-rating Depression Scale (SDS). Chin J Nerv Ment Dis.1986; 12(5): 260.

9. Wang P. Effect of high quality nursing service on anxiety and depression of patients with malignant hematologic diseases. Clin J Chin Med. 2016; 8(21): 31-32.

10. Li Y, Zhang H, Feng Z, Chen S, Liu T, Chen $X$, Tang J, Liao $Y$. Life events, anxiety and depression among doctors and nurses in the emergency department: a study from eleven general hospitals in Hunan province, China. J Psychiat Brain Sci. 2016; 1(1): 2.
11. Golden-Kreutz DM, Thornton LM, Wells-Di Gregorio S, Frierson GM, Jim HS, Carpenter KM, Shelby RA, Andersen BL. Traumatic stress, perceived global stress, and life events: prospectively predicting quality of life in breast cancer patients. Health Psychol. 2005; 24(3): 288-296.

12. Wang LL, Yuan L. Path analysis of resilience and anxiety, depression on quality of life among esophageal carcinoma patients undergoing radiotherapy and chemotherapy. Chin J Pract Nur. 2016; 32(16): 1201-1205.

13. Zhang L, Li Y, Qing Y, Zhu H, Jia G, Li L. The correlation between psychological elasticity and anxiety/depression in patients with cervical cancer after radical operation. J Int Psychiat. 2018; 45(1): 157-159.

14. Ying $L, W u X$, Lin $C$, Jiang L. Traumatic Severity and Trait Resilience as Predictors of Posttraumatic stress Disorders and Depressive Symptoms among Adolescent Survivors of the Wenchuan Earthquake. PLoS One. 2014; 9(2): e89401.

15. Wang $M$, Song $L$, Song $B, D u$, He X, Bai $X$, Duan X, Wang X. Correlation analysis of resilience, defense mechanism and depression in patients with depression. Chin $\mathrm{J}$ Health Psychol. 2017; 25(2): 165-168.

16. Huang $\mathrm{K}, \mathrm{Xu} \mathrm{Q}$, Jiang $\mathrm{M}$, Wang $\mathrm{B}$. Correlation between resilience, anxiety and depression in breast cancer patients receiving postoperative chemotherapy. J Nur Sci. 2013; 28(2): 89-91.

17. Fu TG. Effect of psychological elasticity training and nursing on negativeemotion and anti-stress ability in patients with breast cancer. Psychol Nur. 2017; 21(24): 3242-3244. 